\title{
Valoración de la respuesta endoscópica al tratamiento con argón plasma en la rectitis actínica crónica
}

\author{
M. Latorre Sánchez, J. Sempere García-Argüelles, S. Barceló Cerdá1 , J. M. Huguet Malavés, P. Canelles \\ Gamir, F. Quiles Teodoro y E. Medina Chuliá
}

Servicio de Digestivo. Consorcio Hospital General Universitario de Valencia. 'Departamento de Estadística e Investigación Operativas Aplicadas y Calidad. Universidad Politécnica. Valencia

\section{RESUMEN}

Objetivo: valorar la mejoría endoscópica tras el tratamiento con argón plasma utilizado para el control sintomático de las rectitis actínicas crónicas.

Diseño y pacientes: estudio prospectivo de 48 meses de duración, con 38 pacientes (26 hombres y 12 mujeres, con edad media de 70,9 \pm 7,38 años). Todos presentaban algún grado de rectorragia y/o anemia. Se realizaron entrevistas clínicas, analíticas y sesiones endoscópicas con argón, de forma periódica mensual, hasta la normalización analítica y cese del sangrado, con un seguimiento posterior de 24 meses. Para valorar la mejoría se utilizaron parámetros analíticos, gradación de sangrado (Chutckan) y un índice endoscópico.

Resultados: el tiempo medio trascurrido entre el inicio y la finalización del seguimiento fue de 28,5 $\pm 3,9$ meses. La media de sesiones administradas por paciente ha sido de 3,6 $\pm 2,7$. Si comparamos los síntomas antes, después del tratamiento existe una disminución significativa en el grado de rectorragia según la clasificación de Chutkan $(2,29 \pm 1,08$ vs. 0,59 $\pm 1,12, p<$ $0,05)$. Cuando comparamos los valores medios de la hemoglobina, antes y después del tratamiento apreciamos una aumento estadísticamente significativo $(11,33 \pm 3,05 \mathrm{mg} / \mathrm{dl}$ vs. $14,014 \pm$ $1,29 \mathrm{mg} / \mathrm{dl}, \mathrm{p}<0,001)$ así como los valores medios de ferritina $(31,15 \pm 66,45 \mu \mathrm{g} / \mathrm{dl}$ vs. 80,60 $\pm 55,60 \mu \mathrm{g} / \mathrm{dl}, \mathrm{p}<0,05)$. En el índice endoscópico, también existe una mejoría evidente de la afectación endoscópica tras el tratamiento con respecto a la friabilidad ( $p<0,0001)$ y porcentaje de mucosa afecta $(p<0,0001)$.

Conclusión: el argón plasma es eficaz y la realización del índice endoscópico es una herramienta útil para valorar la mejoría endoscópica de las lesiones tras el tratamiento.

Palabras clave: APC. Argón plasma coagulación. Rectitis actínica crónica. Rectorragia. Anemia.

\begin{abstract}
Objectives: to evaluate endoscopic improvement after argon plasma coagulation (APC) in symptomatic patients with chronic radiation proctopathy.

Methods and patients: a prospective study of 38 patients with radiation proctitis (26 males and 12 females, mean age 70.9 $\pm 7.38 \mathrm{yrs}$ ), and with rectal bleeding and or anemia. We performed monthly interviews, blood tests, and APC sessions until rectal bleeding had ceased and hemoglobin and ferritin levels were improved, with a follow-up of 24 months. We used blood testing, bleeding scores (Chutckhan's index), and endoscopic scores to evaluate improvement.
\end{abstract}

Results: mean time between inclusion and follow-up completion was $28.5 \pm 3.9$ months. Mean number of sessions per patient was $3.6 \pm 2.7$. There was a significant decrease $(2.29 \pm 1.8$ vs. $0.59 \pm 1.12, p<0.05)$ in rectal bleeding (Chutckan score) from baseline after APC. There was a significant increase in hemoglobin levels $(11.3 \pm 3.05$ vs. $14.014 \pm 1.29, p<0.001)$ and ferritin levels $(31.15 \pm 66.45$ vs. $80.60 \pm 55.6, p<0.05)$ from baseline after APC. Also, there was improvement in the endoscopic index at the end of treatment, as well as in friability $(\mathrm{p}<$ $0.0001)$ and involved surface area $(p<0.0001)$.

Conclusion: argon plasma coagulation is an effective technique, and the endoscopic index is a useful tool to evaluate endoscopic improvement.

Key words: APC. Argon plasma coagulation. Chronic radiation proctopathy. Rectal bleeding. Anaemia.

Latorre Sánchez M, Sempere García-Argüelles J, Barceló Cerdá S, Huguet Malavés JM, Canelles Gamir P, Quiles Teodoro F, Medina Chuliá E. Valoración de la respuesta endoscópica al tratamiento con argón plasma en la rectitis actínica crónica. Rev Esp Enferm Dig 2008; 100: 619-624.

Recibido: 03-06-08.

Aceptado: 17-06-08.

Correspondencia: Mercedes Latorre Sánchez. C/ Ovidi Montlor, 5, 4. 46017 Valencia.e-mail: mercedeslatorre@msn.com

\section{INTRODUCCIÓN}

La rectitis actínica crónica es una enfermedad con una incidencia que oscila del 2 al 20\% (1) en los pacientes que han recibido radioterapia como tratamiento de tumo- 
res pélvicos. El tiempo transcurrido entre la aplicación de la radioterapia y la aparición de sintomatología es muy variable, pudiendo oscilar de 3 meses a 31 años. Pese a que el desarrollo de los síntomas suele ser insidioso, las manifestaciones tienden a progresar. Estas manifestaciones suelen cursar con hemorragia o sangrado rectal, siendo la más prevalente (2), apareciendo en el $72 \%$ de los pacientes, tenesmo, diarrea, dolor, estenosis, ulceraciones y fístulas. Acompañándose en ocasiones de alteraciones analíticas con anemia ferropénica.

La medida fundamental del tratamiento es la prevención (3), pero una vez establecida la rectitis se han aplicado diversos tratamientos, desde tópicos con enemas de distintas sustancias (4-8) hasta cirugía (9-11). Gracias al desarrollo de las distintas técnicas endoscópicas (12-19) en el tratamiento de lesiones sangrantes, se ha reducido el número de intervenciones quirúrgicas en la rectitis actínica, quedando limitada su indicación a estenosis, fístulas, necrosis y perforaciones (3).

El argón plasma es el sistema más utilizado en los últimos años por su bajo número de complicaciones (20-23) y por su relativo bajo coste. Es utilizado como sistema de electrocoagulación en las lesiones vasculares. En estudios realizados sobre la eficacia de este método (24-26), los resultados suelen oscilar en torno al $75 \%$ en el control del sangrado.

\section{OBJETIVO}

Valorar la mejoría endoscópica tras el tratamiento de fulguración con argón plasma utilizado para el control sintomático de las rectitis actínicas crónicas.

\section{PACIENTES}

Estudio prospectivo de 48 meses de duración, desde septiembre de 2002 a septiembre de 2006 (periodo de inclusión de septiembre de 2002 a 2004), realizado en la Sección de Endoscopias, Servicio de Digestivo del Consorcio Hospital General Universitario de Valencia. Se incluyeron 38 pacientes consecutivos (26 hombres y 12 mujeres, con edad media de 70,9 $\pm 7,38$ años), procedentes de nuestro centro y del Instituto Valenciano de Oncología. Todos presentaban algún grado de rectorragia y/o anemia y cumplían los criterios de inclusión/exclusión (Tabla I), aceptando su participación y firmando consentimiento informado. El estudio ha sido aprobado por el Comité de Ética del centro.

La aparición de la sintomatología tras la administración de radioterapia (RT) fue de 720,03 $\pm 1.027,44$ días, siendo la dosis media de RT administrada de 62,62 Gy (rango: 14,16-80 Gy). El 39,5\% de los pacientes ya habían llevado un tratamiento previo (enemas de corticoides, 5-ASA, sucralfato), durante 6 a 12 meses, sin mejoría y únicamente 3 pacientes $(7,9 \%)$ habían padecido
Tabla I. Criterios de inclusión/exclusión

\begin{tabular}{lc}
\hline Criterios de inclusión & Criterios de exclusión \\
\hline $\begin{array}{l}\text { Diagnóstico endoscópico altamente compatible } \\
\text { (patrón vascular característico, telangiectasias) } \\
\text { con rectitis actínica y: }\end{array}$ & $\begin{array}{c}\text { - Lesiones endoscópicas dudosas o } \\
\text { coexistencia de otras lesiones causantes } \\
\text { de rectorragia o anemia (inflamatoria, } \\
\text { vascular o neoplásica) }\end{array}$ \\
- Rectorragia o anemia (ferropénica) & - Asociar enfermedades potencialmente \\
& anemizantes (cirrosis, VIH, enfermedad \\
- Tiempo finalización radioterapia (RT) e & - Ausencia de rectorragia y anemia. \\
inclusión estudio > 12 meses & - Intervalo de RT e inclusión inferior a 12 \\
- Curación completa tumor primitivo & meses \\
(que motivó RT) & - Cualquier lesion neoplásica y/o \\
- Aceptación mediante consentimiento & metástasis \\
informado &
\end{tabular}

además toxicidad gastrointestinal aguda. Además, el $34,2 \%$ de los pacientes tomaban tratamiento anticoagulante o antiagregante. En 23 pacientes fue necesaria la administración de suplementos de hierro oral y a 9 pacientes se les administró también transfusión sanguínea.

\section{MÉTODOS}

Se realizaron entrevistas clínicas, analíticas y sesiones endoscópicas con argón, de forma periódica mensual, hasta la normalización analítica y cese del sangrado con un máximo de 10 sesiones endoscópicas. Tras finalizar las sesiones, se realizó un seguimiento clínico-analítico de 24 meses (a los 2, 6, 12 y 24 meses).

La valoración de la mejoría del sangrado se define como disminución en al menos un grado de rectorragia tras el tratamiento, utilizándose la clasificación de Chutckan (19) (Tabla II). Los datos del hemograma, ferritina y sideremia, se han utilizado para valorar mejoría analítica; para establecer la mejoría endoscópica (disminución del número de telangiectasias en la mucosa del colon) se ha empleado un índice de afectación que debe ser valorado en todas las sesiones por al menos dos endoscopistas, en el que se constata la localización, longitud en centímetros, porcentaje de la circunferencia de la mucosa del colon afecta y friabilidad de las lesiones. Todas las sesiones endoscópicas se han grabado en vídeo. Se define como el éxito del tratamiento el cese del sangrado y la normalización de los parámetros analíti$\cos$.

Tabla II. Gradación de la rectorragia (clasificación de Chutckan)

\begin{tabular}{ll}
\hline Grado 0 & No \\
\hline Grado 1 & Rectorragia escasa en el papel al limpiarse \\
Grado 2 & Sangrado en la taza de WC \\
Grado 3 & Abundante sangrado incluso manchando la ropa \\
Grado 4 & Sangrado con necesidad transfusional
\end{tabular}


Tabla III. Clasificación de afectación endoscópica antes y después del tratamiento

\begin{tabular}{|c|c|c|c|}
\hline & Antes & Después & Resultados estadisticos \\
\hline Segmento afecto & $\begin{array}{l}\text { Recto } 23 p(60,5 \%) \\
\text { Recto-sigma } 13 p(34,2 \%) \\
\text { Descendente-S-R } 2 \text { p }(5,3 \%)\end{array}$ & $\begin{array}{l}\text { Recto } 23(60,5 \%) \\
\text { Recto-sigma } 13(34,2 \%) \\
\text { Descendente-S-R } 2(5,3 \%)\end{array}$ & \\
\hline Longitud & $13,97 \pm 10,11$ & $19,67 \pm 10,68$ & $\begin{array}{l}t \text { Student datos apareados } \\
p=0,317 \\
\text { No significativo }\end{array}$ \\
\hline Friabilidad & $\begin{array}{l}\text { Grado 0: sin friabilidad, } 7 p \\
\text { Grado 1: friabilidad al roce, } 15 p \\
\text { Grado 2: friabilidad espontánea, } 10 p \\
\text { Grado 3: Hemorragia profusa, } 6 p\end{array}$ & $\begin{array}{l}\text { Grado 0: sin friabilidad, } 30 p \\
\text { Grado 1: friabilidad al roce, } 8 p \\
\text { Grado 2: friabilidad espontánea, } 0 p \\
\text { Grado 3: Hemorragia profusa, } 0 p\end{array}$ & $\begin{array}{l}\text { Test de Wilcoxon } \\
p=0,0002 \\
\text { Significativo }\end{array}$ \\
\hline
\end{tabular}

p: pacientes.

La preparación intestinal del paciente constaba de dieta líquida y toma de solución comercial oral de fosfato de sodio. Las sesiones endoscópicas se realizaban con una fuente de diatermia (ERBE ICC 200, Medtech Systems, Adelaida South Australia) con flujos entre 1,4-1,8 1/min, potencias entre 50-60 vatios y una duración de 15-20 minutos. Se aplicó la técnica de punteado comenzando inicialmente de tramos proximales a distales con aspiraciones frecuentes.

Los datos recogidos se introdujeron en la base de datos que se realizó para tal fin utilizando el programa SPSS versión 12.5, para elaborar el análisis estadístico. Se utilizó el test de Wilcoxon para las variables endoscópicas y gradación del sangrado. Para las variables analíticas se utilizó test de Student para datos pareados y para valorar la variabilidad interobservador en la aplicación del índice endoscópico se utilizó el coeficiente kappa. Se utilizó el coeficiente de correlación de Pearson o Spearman, según se tratase de datos cuantitativos o no, para relacionar el número de sesiones necesarias con las variables del índice endoscópico inicial.

\section{RESULTADOS}

El tiempo medio trascurrido entre el periodo de inclusión (antes del tratamiento) y la finalización del seguimiento (después del tratamiento) fue de 28,5 $\pm 3,9$ meses, siendo la media de las sesiones administradas por paciente de $3,6 \pm 2,7$.

El grado de rectorragia de los pacientes tras el tratamiento y seguimiento durante 24 meses, ha disminuido de forma estadísticamente significativa cuando lo comparamos con los datos iniciales $(2,29 \pm 1,08 v s .0,59 \pm$ $1,12, \mathrm{p}<0,05)$. Cuando comparamos los valores medios de la hemoglobina antes y después del tratamiento, apreciamos un aumento estadísticamente significativo $(11,33 \pm 3,05 \mathrm{~g} / \mathrm{dl} v s .14,014 \pm 1,29 \mathrm{~g} / \mathrm{dl}, \mathrm{p}<0,001)$, así como en los niveles de ferritina $(31,15 \pm 66,45 \mu \mathrm{g} / \mathrm{dl}$ vs. 80,60 $\pm 5,60 \mu \mathrm{g} / \mathrm{dl}, \mathrm{p}<0,05)$. Ningún paciente ha vuelto a precisar administración de suplementos de hierro ni transfusiones sanguíneas y los resangrados, tras la finalización del tratamiento, aparecieron a partir de los 6 primeros meses (media $28 \pm 12$ semanas) durante el seguimiento en 5 pacientes $(13,15 \%)$.

También existe mejoría estadísticamente significativa en la afectación endoscópica tras el tratamiento (Tabla III). Cuando comparamos la friabilidad (Fig. 1) y el porcentaje de circunferencia mucosa afecta (Fig. 2), antes y después del tratamiento (Fig. 3), existe una dismi-

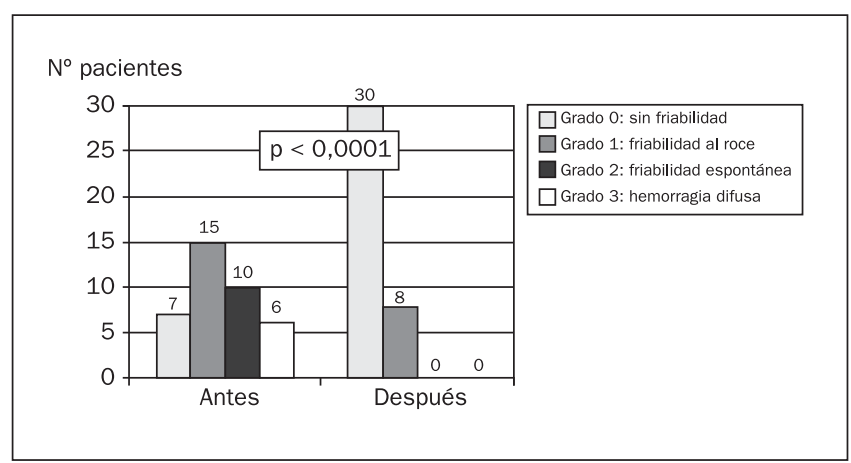

Fig. 1. Comparación de la friablidad de la mucosa afecta antes y después del tratamiento.

$\mathrm{N}^{\circ}$ pacientes

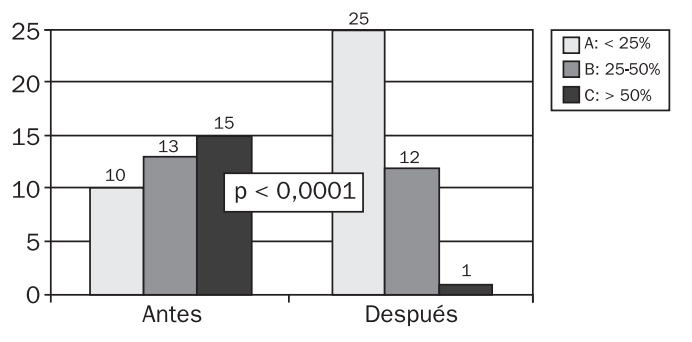

Fig. 2. Comparación del porcentaje circunferencial de mucosa afecta antes y después del tratamiento. 

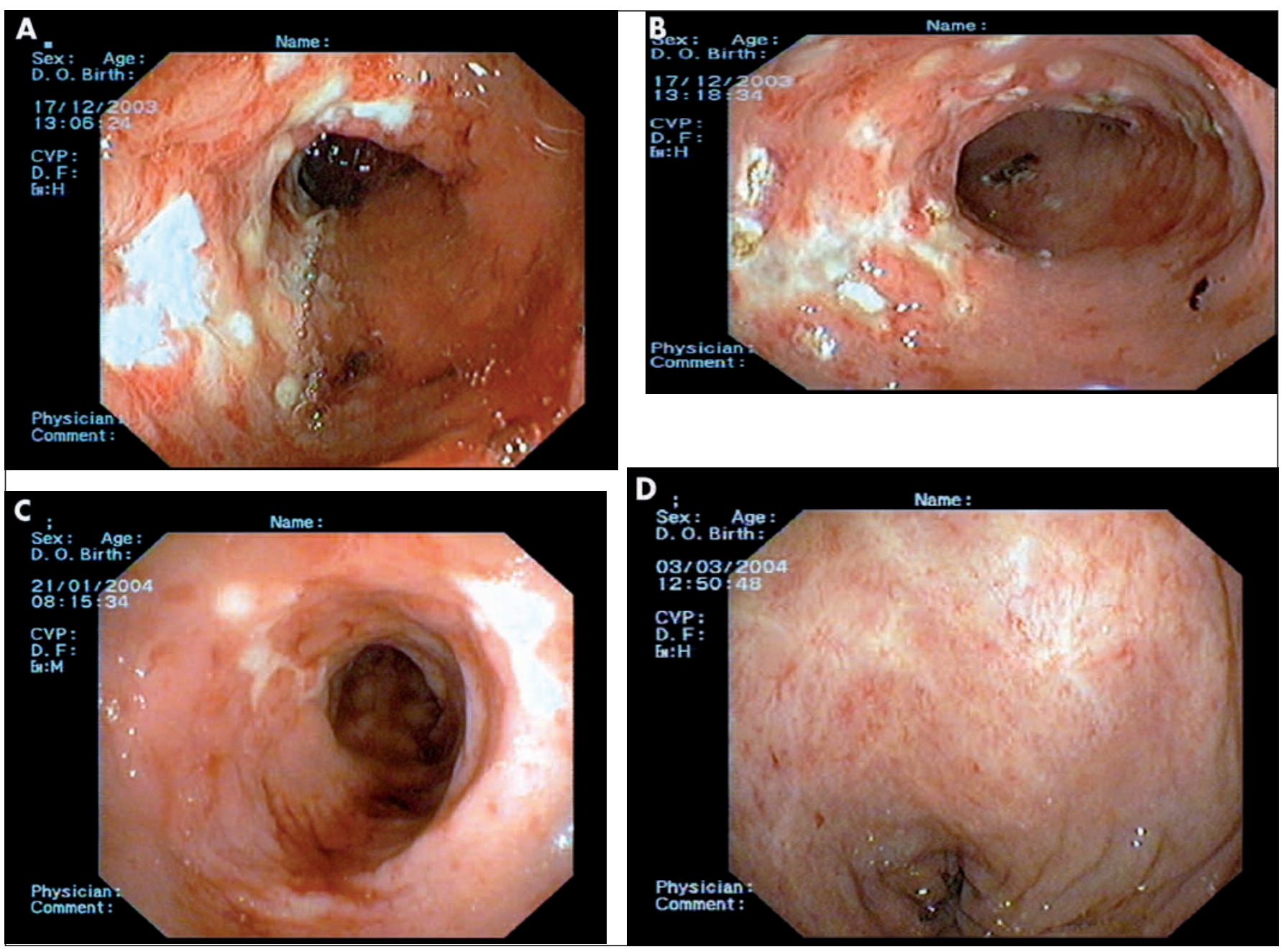

Fig. 3. Mejoría endoscópica del paciente $n^{\circ} 5$.

nución en la gradación de ambas (test de Wilcoxon), con $\mathrm{p}<0,0001$. La disminución de la longitud no fue significativa $(p=0,3)$. La concordancia interobservador en la descripción de las lesiones utilizando la gradación endoscópica fue de $0,7(\mathrm{p}=0,0002)$, según el índice kappa.

El número de sesiones endoscópicas requeridas se correlacionaba de forma estadísticamente significativa con la severidad endoscópica de las lesiones (Tabla IV).

No hubo ninguna complicación en el 76,76\% de las sesiones (109/142). Las complicaciones mayores fueron rectorragia con necesidad de ingreso hospitalario en el $1,4 \%$ de las sesiones (2/142), y las complicaciones leves que aparecieron en el 23,23\% de las sesiones (33/142) fueron: proctalgia postsesión en 7,9\% de las sesiones; rectorragia mayor a la habitual, en $23,7 \%$ de las sesiones y febrícula en $2,6 \%$.

Se ha conseguido el éxito del tratamiento en el 86,8\%, de los pacientes.
Tabla IV. Correlación entre el número de sesiones y datos endoscópicos iniciales

\begin{tabular}{ccccc}
\hline & Segmento afecto & Longitud $(\mathrm{cm})$ & Porcentaje de mucosa & Friabilidad \\
\hline$N^{\circ}$ sesiones & 0,442 & 0,455 & 0,715 & 0,715 \\
& $\begin{array}{c}\text { (rho de) } \\
\text { Spearman }\end{array}$ & $\begin{array}{c}\text { (coeficiente } \\
\text { de Pearson) }\end{array}$ & $\begin{array}{c}\text { (rho de } \\
\text { Spearman) }\end{array}$ & $\begin{array}{c}\text { Spearman) } \\
\text { Sho de }\end{array}$ \\
$\begin{array}{cccc}0,005 \\
\text { Significativo }\end{array}$ & $\begin{array}{c}0,004 \\
\text { Significativo }\end{array}$ & $\begin{array}{c}0,000 \\
\text { Significativo }\end{array}$ & $\begin{array}{c}0,000 \\
\text { Significativo }\end{array}$ \\
\hline
\end{tabular}

\section{DISCUSIÓN}

El argón plasma es un método eficaz y relativamente seguro para el control de la rectorragia y/o anemia que se produce en la rectitis actínica crónica, tal y como han mostrado las diversas series publicadas (22-25), aunque con menor número de pacientes. Todos coinciden en la mejoría sintomática de la rectorragia, oscilando entre el 80 y el 99\% (23-26). 
Tanto la edad, como las dosis de radioterapia de nuestros pacientes no difieren de otras series (26-28). En 15 pacientes el tratamiento con argón se había administrado tras el fracaso de tratamientos tópicos utilizados durante un largo periodo de tiempo, al igual que otros trabajos publicados $(25,27)$. El 34,2\% de los pacientes tomaban tratamiento anticoagulante o antiagregante, aumentando la friabilidad de las lesiones, pese a su retirada anterior a la realización de las sesiones endoscópicas terapéuticas $(29,30)$.

En nuestra serie, todos los pacientes presentaban una disminución de al menos un grado en la escala de sangrado tras el tratamiento, con respecto al periodo de inclusión, y sólo en el $86,8 \%$ se ha conseguido el cese total del sangrado. Existen pocos artículos en los que se diferencie el cese total del sangrado de la disminución $(24,25)$, siendo el porcentaje de cese del sangrado inferior al nuestro. Los resangrados aparecieron en 5 pacientes durante el periodo de seguimiento, en grado 1. A todos ellos se les realizó una nueva colonoscopia con sesión endoscópica si precisasen, fulgurándose en 3 pacientes pequeñas telangiectasias residuales, cercanas al margen anal, que no se habían tratado inicialmente y continuando posteriormente el seguimiento clínico-analítico durante 24 meses más, sin complicaciones posteriores. Atribuimos los resangrados a dos circunstancias, la técnica endoscópica utilizada, puesto que eran los primeros pacientes que se habían tratado y en los que dejamos mayor longitud sin tratar de lesiones cercanas al margen anal y además todos ellos presentaban hemorroides internas (24). Quizás debimos desestimar a todos los pacientes con hemorroides internas, aunque únicamente incluimos $\operatorname{los}$ grados 1 y 2 , pero debido a su presentación común y su baja frecuencia de anemización, no lo realizamos.

En la mayoría de trabajos (23-25,28), los parámetros analíticos se basan únicamente en la hemoglobina sérica o el hematocrito, con aumentos en sus niveles medios tras la finalización del tratamiento que oscilan entre 3 y 4 $\mathrm{mg} / \mathrm{dl}$, coincidentes con los obtenidos en nuestra serie. No existen datos de los niveles de sideremia sérica ni ferritina de los pacientes en otros artículos, por lo que no podemos comparar nuestros resultados, aunque sí coincidimos en la ausencia tanto de transfusiones como del uso del hierro oral tras el tratamiento $(26,28)$.

El índice endoscópico, poco utilizado hasta el momento, es una herramienta útil en la gradación de las lesiones. Coincidimos con el artículo de Canard y cols. (31) donde utilizan un índice endoscópico similar al nuestro en la friabilidad de las lesiones, además de la longitud que hasta el momento era el único parámetro endoscópico utilizado. En su estudio retrospectivo incluyen 30 pacientes con seguimiento no estandarizado de 3 a 35 meses, utilizando un índice endoscópico para valorar la friabilidad de la mucosa en cuatro grados, al inicio y tras la finalización de las sesiones endoscópicas con argón, pasando de un score inicial de 1,61 a 0,3 ( $\mathrm{p}<0,002)$, similar a nuestros resultados (de 1,39 a 0,21; p < 0,002). Zinicola y cols. (32) y posteriormente Chi y cols. (33), con estudios retrospectivos, incorporan también en sus estudios índices endoscópicos basados en la distribución de las telangiectasias, porcentaje de mucosa afecta y la presencia o no de sangre fresca; realizando una escala en tres grados muy útil pero sin incluir una gradación de friabilidad de la mucosa, parámetro importante en la valoración de la mejoría, tal y como se muestra en el trabajo de Canard y en el nuestro. Quizás lo óptimo sería unificar criterios utilizando un único índice.

En cuanto a la media de sesiones necesarias para la remisión, está directamente correlacionada con la severidad de las lesiones endoscópicas, sobre todo con la friabilidad y porcentaje de mucosa afecta, coincidiendo con otros resultados publicados (26). Pese a que nuestro objetivo no fue tratar todas las lesiones accesibles, sino las necesarias hasta que cediesen los síntomas y hubiese normalización de los parámetros analíticos, nuestra media de sesiones es ligeramente superior a otras series, pudiendo corresponder a la limitación del tiempo por sesión, inferior a 30 minutos, para reducir las complicaciones, así como a la técnica de fulguración en forma de punteado, con aspiraciones frecuentes.

Nuestra media de complicaciones leves es ligeramente inferior a otros autores que utilizan flujos inferiores a $2 \mathrm{l} / \mathrm{min}$ y potencias inferiores a 60 vatios $(28,29,34)$, quizás porque pese a limitar estos parámetros, no han limitado el tiempo de las sesiones.

Creemos por tanto, que el argón es un método útil en el control de la rectorragia producida en esta enfermedad y debemos utilizar flujos inferiores a $2 \mathrm{l} / \mathrm{min}$, con potencias inferiores a 60 vatios, no superando los 30 minutos por sesión, si queremos limitar las complicaciones. Así mismo, la gradación de las lesiones utilizando un índice endoscópico es importante para valorar la mejoría y poder realizar un pronóstico, para aproximarnos en el número de sesiones terapéuticas necesarias para la remisión.

\section{CONCLUSIÓN}

El argón plasma es eficaz como tratamiento en el control sintomático de la anemia y/o rectorragia, derivadas de la rectitis actínica crónica, con bajas complicaciones. La realización del índice endoscópico es una herramienta útil para valorar la mejoría endoscópica de las lesiones y el número de sesiones necesarias para su remisión.

\section{AGRADECIMIENTOS}

Agradecemos la ayuda prestada en la inclusión de pacientes al Servicio de Radioterapia del Consorcio Hospital General Universitario de Valencia, especialmente al Dr. López-Torrecillas y al Instituto Valenciano de Oncología. 


\section{BIBLIOGRAFÍA}

1. Babb RR. Radiation proctitis: a review. Am J Gastroenterol 1996; 91 : 1309-11.

2. Jackson A. Partial irradiation of the rectum. Semin Radiat Oncol 2001; $11: 215-23$

3. Johnston MJ, et al. Management of late complications of pelvic radiation in the rectum and anus. Dis Colon Rectum 2003; 46: 247-59.

4. Baum CA, Biddle WL. Failure of 5-animosalicylic acid enemas to improve chronic radiation proctitis. Dig Dis Sci 1989; 34: 758-60.

5. Stockdale AD, Biswas A. Long-term control of radiation proctitis following treatment with sucralfate enemas. Br J Surg 1997; 84: 379 (abstract).

6. Talley NA, Chen F, King D, et al. Short chain fatty acids in the treatment of radiation proctitis: a randomised, double blind, placebo-controlled, crossover pilot trial (abstract). Dis Colon Rectum 1997; 40: 1046-9.

7. Pinto A, Hidalgo P, Cravo M, Nobre Leitao C. Short chain fatty acids are effective in short-term treatment of chronic radiation proctitis. Dis Colon Rectum 1999; 42: 788-95.

8. Vernia P, Fracasso PL, Casale V. Topical butyrate for acute radiation proctitis: randomised, crossover trial. Lancet 2000; 356: 1232-5.

9. Kimose HH, Fisher L, Spjednaes N, et al. Late radiation injury of the colon and rectum surgical management and outcome. Dis Colon Rectum 1989; 32: 684-9.

10. Lucarotti ME, Mouuntford RA, Bartolo DC. Surgical management of intestinal radiation injury. Dis Colon Rectum 1991; 34: 865-9.

11. Von Flue MO, Degen LP, Beglinger C, Harder FH. The ileocecal reservoir for rectal replacement in the complicated radiation proctitis. Am J Surg 1996; 172: 335-40.

12. Da Paredes V, Bauer P, Bourguignon J, Sultans S, Thomas C, Mory $\mathrm{B}$, et al. Formalina dans les rectites radiques chroniques hémaorragiques: résultats d'une étude prospective chez 21 pacientes. Gastroenterologie Clinique et Biologique 2000; 24: A34.

13. Pikarsky AJ, Belin B, Efron J, et al. Complications following formalin installation in the treatment of radiation induced proctitis. Int J Colorectal Dis 2000; 15: 96-9.

14. Luna-Pérez P, Rodríguez-Ramírez SE. Formalin instillation for refractory radiation-induced hemorrhagic proctitis. J Surg Oncol 2002; 80: 41-4.

15. Cullen SN, Frenz M, Mee A. Treatment of haemorrhagic radiation-induced proctopathy using small volume topical formalin instillation. Alimentary Pharmacology \& Therapeutics 2006; 23: 1575-80.

16. Vyas FL, Mathai V, Selvamani B, et al. Endoluminal formalin application for haemorrhagic radiation proctitis. Colorectal Disease 2006; 8: $342-6$

17. Viggiano TR, Zighelboim J, Ahlquist DA, Goustout $\mathrm{Cj}$, Wang KK, Larson MV. Endoscopic ND: Yang laser coagulation of bleeding from radiation proctopathy. Gastrointest Endosc 1993; 39: 513-7 (abstract).
18. Jensen DM, Machicado GA, Cheng S, et al. A randomized prospective study of endoscopic bipolar electrocoagulation and heater probe treatment of chronic rectal bleeding from radiation tealangiectasia. Gastrointest Endosc 1997; 45: 20-5 (abstract).

19. Chutkan R, Lipp J, Waye J. The argon plasma coagulator: a new and effective modality for the treatment of radiation proctitis (abstract). Gastrointest Endosc 1997; 45: AB27.

20. Fantin AC, Binek j, Suter Wr, Meyerberger C. Argon beam coagulation for treatment of symptomatic radiation-induced proctitis. Gastrointest Endosc 1999; 49: 515-8.

21. Silva RA, Correia AJ, Dias LM, et al. Argon plasma coagulation for hemorrhagic radiation proctosigmoiditis. Gastrointest Endosc 1999 50: 221-4.

22. Tam W, Moore J, Schoeman M. Treatment of radiation proctitis with argon plasma coagulation. Endoscopy 2000; 32 (9): 667-72.

23. Kaassis M, Oberti F, Burtin P, Boyer J. Argon plasma coagulation for the treatment of hemorrhagic radiation proctitis. Endoscopy 2000; 32 (9): 673-6.

24. Villavicencio RT. Efficacy and complications of argon plasma coagulation for hematochezia related to radiation proctopathy. Gastrointest Endosc 2002; 55 (1): 70-4

25. Tjandra JJ, Sengupta S. Argon plasma coagulation is an effective treatment for the refractory hemorrhagic radiation proctitis. Dis Colon Rectum 2001; 44: 1759-65.

26. Taïeb S, et al. Effective use of argon plasma coagulation in the treatment of the severe radiation proctitis. Dis Colon Rectum 2001; 44 1766-71.

27. Rotondano G, Bianco MA, Marmo R, Piscopo R, Cipolleta L. Longterm outcome of argon plasma coagulation for bleeding caused by chronic radiation proctopathy. Dig Liver Dis 2003; 35: 806-10.

28. Ravizza D, Fiori G, Torvato C, Crosta C. Frequency and outcomes of ulcers during argon plasma coagulation for chronic radiation induced proctopathy. Gastrointest Endosc 2003; 57: 519-25.

29. ASGE. American Society of Gastrointestinal Endoscopy. Guidelines on the management of anticoagulation and antiplatelet therapy for endoscopic procedures. Gastrointest Endosc 2002; 55: 775-9.

30. ASGE Guideline: the management of low-molecular-weight heparin and nonaspirin agents for endoscopic procedures. Gastrointest Endosc 2005; 61: 189-94.

31. Canard JM, Vedrene B, Bors G, Clauce P, Bader R, Sonedag D. Treatment of radiation proctitis by coagulation: long-term results Gastroenterologie Clinique et Biologique 2003; 27: 455-9.

32. Zinicola R, Rutter MD, Falasco G, Brooker JC, Cennamo V, Contini $\mathrm{S}$, et al. Haemorrhagic radiation proctitis: endoscopic severity may be useful to guide therapy. Int J Colorectal Dis 2003; 18: 439-44.

33. Chi KD, Ehrenpreis ED, Jani AB. Accuracy and rehability of the endoscopic classification of chronic radiation induced proctopathy using a novel method. J Clin Gastroenterol 2005; 39 (1): 42-6.

34. Postgate A, Saunders B, Tjandra J, Vargo J. Argon plasma coagulation in chronic radiation proctitis. Endoscopy 2007; 39 (4): 361-5. 